\title{
Valores objeto de conservación del subsistema de áreas protegidas de los Andes occidentales, Colombia
}

\author{
(1) Néstor Javier Roncancio-Duque*, ㄴ) Laura Alejandra Vélez Vanegas \\ Dirección Territorial Andes Occidentales, Parques Nacionales Naturales de Colombia, Bogotá, D.C., Colombia
}

\begin{abstract}
Resumen
Para planificar el manejo de la conservación de la biodiversidad y de los recursos naturales se deben formular objetivos específicos y medibles que permitan evaluar la efectividad de la gestión. Su formulación específica para cada uno de los componentes de la biodiversidad o de los servicios ecosistémicos derivados de sus procesos ecológicos no es práctico, pues ello no ayuda a una gestión ambiental efectiva. Por lo tanto, se deben seleccionar unos elementos sustitutos que representen los objetivos de la conservación y las situaciones de manejo que deben resolverse para alcanzarlos. En este sentido, como paso inicial para revisar la planeación del manejo de todo el territorio, el equipo del Subsistema de Áreas Protegidas Andes Occidentales (SAO) llevó a cabo la selección de esos elementos sustitutos denominados "valores objeto de conservación" usando el enfoque de especies del paisaje, el cual permite definir un grupo de especies animales que cumplen funciones de especies sombrilla, piedra angular y bandera. En el SAO se definieron nueve especies, entre las cuales se encuentran cuatro carnívoros, incluidos dos felinos, mamíferos acuáticos, un ave, un ungulado, un primate y un roedor. Con ello se aspiraba a generar un plan de manejo concertado con todos los actores del territorio para lograr su conservación efectiva y consolidar la estructura ecológica del subsistema. (C) 2019. Acad. Colomb. Cienc. Ex. Fis. Nat.
\end{abstract}

Palabras clave: Objetivos de conservación; Especies de paisaje; Manejo efectivo.

Values of conservation objects in the West Andean protected areas subsystem

\begin{abstract}
In order to plan the conservation management of the biodiversity and of natural recourses in general, we must formulate specific and measurable goals to assess how effective such management is. It is not practical to define such goals for each component of the biodiversity and the ecosystem services derived from the ecological process, as this does not allow for an effective environmental management. Thus, it is necessary to select some surrogate items representing the conservation goals and the management situations that must be resolved to achieve them. In this sense, and as an initial step to review and fit its management plan to the whole territory, the West Andean protected areas subsystem (SAO) team selected those surrogate items and called them values of the conservation objects. The selection was done using the Landscape Species Approach to define a group of animals as umbrella, keystone and flagship species. We selected nine species: four carnivorous including two felids, as well as aquatic mammals, a bird, an ungulate, a primate, and a rodent. With this, we expected to come up with management plans based on the cooperation with all relevant stakeholders in the territory for an effective conservation effort aimed at consolidating the ecological structure of this subsystem. (C) 2019. Acad. Colomb. Cienc. Ex. Fis. Nat.
\end{abstract}

Key words: Object of conservation; Landscape species; Effective management.

\section{Introducción}

Mediante la gestión ambiental se busca conservar toda la biodiversidad en un territorio o, por lo menos, dentro de una estructura ecológica que permita mantener la oferta de servicios ecosistémicos en un paisaje determinado. No obstante, no es eficiente planear e implementar estrategias de conservación para cada uno de los componentes de la diversidad biológica (Roberge \& Angelstam, 2004). Por lo tanto, se hace necesario seleccionar una serie de elementos sustitutos que representen las situaciones de manejo (objetivos de conservación, amenazas y causas en el paisaje), en torno a las cuales se puedan formular objetivos medibles y llevar a cabo un monitoreo efectivo que permita adaptar las acciones de manejo en el marco de un proceso de adopción estructurada de decisiones (Holling, 1978; Lyons, et al., 2008). La pretensión es que, al lograr un escenario de conservación efectivo para los objetos sustitutos, se conserve toda la biodiversidad que está en simpatría con ellos (Marcot \& Flather, 2007).

\footnotetext{
*Correspondencia:

Nestor Javier Roncancio Duque; monitoreo.dtao@parquesnacionales.gov.co

Recibido: 9 de agosto de 2018

Aceptado: 14 de febrero de 2019

Editor: Jaime Cantera
} 
Las autoridades ambientales y entidades de apoyo, como las ONG y la academia, hacen ejercicios de selección de objetos sustitutos, los cuales reciben el nombre de objetos de conservación o valores objeto de conservación en algunos escenarios. Algunas veces, tácitamente la selección se apega a conceptos como especie sombrilla (Branton \& Richardson, 2011; Fleishman, et al., 2000; Roberge \& Angelstam, 2004; Wilcox, 1984), especie focal (Lambeck, 1997) o especie piedra angular (keystone) (Caro \& O'Doherty, 1999). Sin embargo, en la mayoría de los casos la selección se hace bajo un criterio de rareza de la especie, es decir, aquellas con distribución restringida (endémicas) o bajas densidades, y en otros casos se limita únicamente a un criterio de carisma, especies bandera (Sergio, et al., 2006; Western, 1987) vulnerabilidad o, simplemente, a oportunidades de gestión, confundiendo, muchas veces, especies indicadores con especies objetivo (Caro \& O'Doherty, 1999; Feinsinger, 2001). En cualquier caso, la selección de los sustitutos no responde a un criterio ecológico (Andelman \& Fagan, 2000; Lindenmayer, et al., 2014; Sergio, et al., 2008), no está justificada con referentes cuantitativos bien definidos y, por ello, puede estar permeada por el sesgo de los participantes en el proceso (Jarro-Fajardo, 2011; Nekaris, et al., 2015; Stevens, et al., 2011; Zambrano, 2010).

Estas debilidades metodológicas tienen consecuencias que se reflejan en un gran número de elementos sustitutos redundantes para un mismo paisaje, los cuales en muchos casos no están ligados de manera directa a ninguna situación de manejo. Es así como los planificadores de la conservación se ven abocados recurrentemente a la formulación de planes de manejo de especies en los que se repiten las estrategias de intervención para un mismo paisaje sin objetivos espacialmente explícitos (Angelstam, et al., 2003; Sanderson, et al., 2002), lo que resulta en planes de manejo muy generales cuya implementación no es efectiva y, si lo llega a ser, no contribuye a tener un sistema eficazmente gestionado (Rondinini, et al., 2011).

La consecuencia de esta selección sin justificación ecológica es la dificultad para determinar los indicadores adecuados y establecer planes de monitoreo ajustados a la planificación de las acciones de manejo (Rondinini, et al., 2011). En este sentido, atendiendo a la concepción generalizada de que la investigación transversal es necesaria para la conservación, se hacen ingentes esfuerzos para la recolección de datos que no se enfocan en resolver los vacíos de información requerida para el manejo, con lo cual los recursos disponibles se desperdician (Caro \& O'Doherty, 1999; Sergio, et al., 2008).

En este contexto, y en aras de revisar y actualizar la planificación para la conservación de su territorio de acción, el equipo del Subsistema de Áreas Protegidas de los Andes Occidentales (SAO) llevó a cabo el proceso de selección de valores objeto de conservación en la perspectiva de resolver los aspectos metodológicos deficientes para así hacer una selección mínima y complementaria (no redundante) de elementos sustitutos de la biodiversidad y de los servicios ecosistémicos. En dicha selección se recurrió al enfoque de especies paisaje (Coppolillo, et al., 2004), en el cual se selecciona un conjunto de especies animales a las que se les puede determinar un rango de hogar y de necesidades de dispersión individual (Baguette, et al., 2013) con base en criterios de heterogeneidad y área mínima para satisfacer su requerimientos ecológicos, la vulnerabilidad local frente a las actividades humanas, la funcionalidad ecológica (Sattler, et al., 2014) y el reconocimiento e interacción con las poblaciones humanas (Coppolillo, et al., 2004). Este tipo de selección de objetos sustitutos ecológicamente justificada combina de manera ponderada las características de las especies sombrilla, focal, piedra angular y bandera (Rasmussen, 2014).

\section{Materiales y métodos}

Área de estudio. La Dirección Territorial Andes Occidentales de Colombia abarca un área de 15.844.104 hectáreas distribuidas en 188 ecosistemas que van desde zonas arenosas y ríos hasta bosques de páramo pluvial en orobiomas diferenciados para cada vertiente de las cordilleras, zonas nivales y afloramientos rocosos (Ríos-Franco \& Valencia, 2014). Este paisaje conserva cerca de 6,67 millones de hectáreas ( $42 \%$ ) de bosques y áreas seminaturales, mientras que los territorios agrícolas ocupan alrededor de 8,88 millones de hectáreas (56 \%). Aproximadamente 200.000 hectáreas son áreas húmedas y superficies de agua naturales y artificiales. El uso del suelo está principalmente asociado a ganadería extensiva (pastos), cultivos de café, caña de azúcar y arroz, y cerca de 600 centros urbanos que demandan los servicios ecosistémicos de las áreas de conservación (Figura 1).

Selección de los valores objeto de conservación (VOC). Para la selección de los VOC de la Dirección Territorial se empleó el método de especies paisaje (Landscape Species Approach), con la aproximación conceptual propuesto en los boletines 2, 3 y 4 del Living Landscapes Program de la Wildlife Conservation Society (WCS) (2001-2002) y el manual técnico 5 de este mismo programa (Stringberg, 2007). El objetivo inicial del método es elegir un conjunto limitado de especies animales a cuyos individuos se les pueden definir valores de dispersión y que puedan representar todos los objetivos de conservación definidos para el paisaje y sirvan para monitorizar la efectividad de las estrategias implementadas (Elzinga, et al., 2001; Sergio, et al., 2008). La selección de los valores objeto de conservación, o especies paisaje, se basó en cinco criterios: 1) el área, es decir, las especies que necesiten mayor cantidad de área para sostener poblaciones viables; 2) la heterogeneidad, o sea las especies que requieren más tipos de cobertura vegetal, o asociaciones vegetales naturales y zonas de manejo (diferentes tipos de aproximación para el manejo, por ejemplo, la de las jurisdicciones administrativas), para satisfacer sus requerimientos ecológicos; 3) la vulnerabilidad, es decir, las especies que hoy sufren el impacto, o pueden sufrirlo 

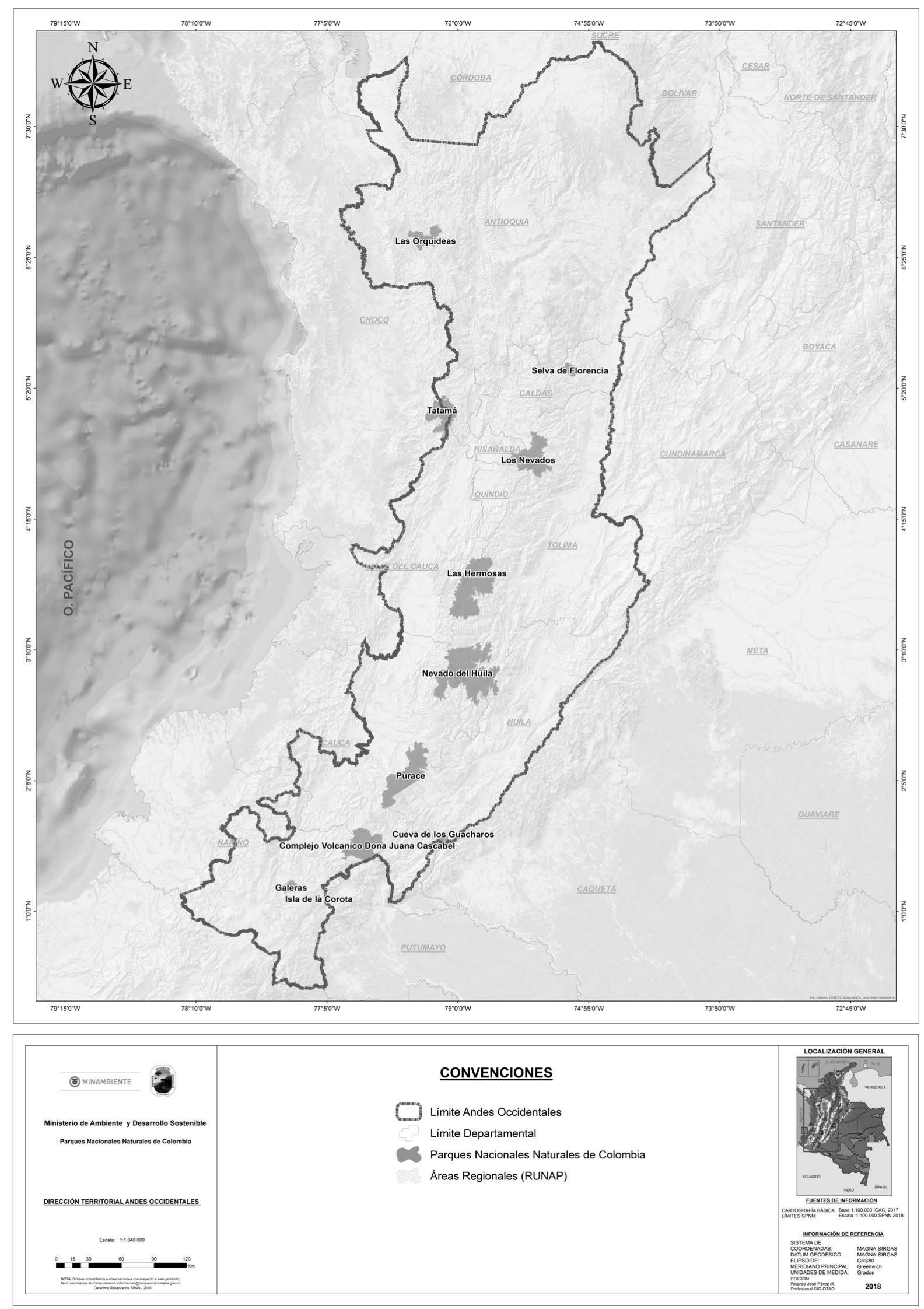

Figura 1. Subsistema de los Andes Occidentales, Colombia 
en el futuro, de las actividades humanas en términos de la gravedad, la reversibilidad y la proporción del área afectada; 4) la funcionalidad ecológica, o sea, las especies que cumplen más funciones dentro del ecosistema (polinización, degradación de materia orgánica, depredación, exclusión competitiva, etc.), y 5) la importancia socioeconómica, referida a las especies que, en términos culturales o económicos, tengan mayor significación positiva o negativa $\mathrm{y}$ puedan, además, ser especies bandera o carismáticas. Las especies seleccionadas fueron aquellas que cumplieron de manera más ajustada con estos criterios.

En este sentido, fue necesario contar con los parámetros y los criterios de selección. Los primeros recogen la información acerca del paisaje en el cual se va a hacer la selección de los valores objeto de conservación. En este caso, se referían al número de ecosistemas, biomas o tipos de asociaciones vegetales del paisaje, los tipos de zonas de manejo, el área que ocupan, las actividades humanas que se dan en el paisaje y que sean, o puedan llegar a convertirse en una amenaza para la conservación de la biodiversidad y los servicios ecosistémicos. Asimismo, se decidió recoger la información sobre el rango de hogar con datos continuos. Además, se determinó cuantitativamente el momento en que se podía considerar que un tipo de cobertura, ecosistema o zona de manejo estaba representado por una especie. Por último, se relacionaron las funciones ecológicas presentes en el paisaje y se definió a partir de qué valor se podía considerar que las representaba una especie.

En cuanto a los criterios de selección, se propuso inicialmente una serie de especies candidatas que cumplieran con uno o más de los criterios. Con base en los criterios definidos tienden a seleccionarse especies de vertebrados grandes, mamíferos, aves o reptiles producto de la relación alométrica entre el tamaño corporal y el rango de hogar (Sinclair, 2003; Wilcox, 1984), y la relación positiva entre la masa y la distancia de dispersión (Jenkins, et al., 2007). Pensando en las oportunidades de gestión, las cuales son mayores para las especies que se encuentran en alguna categoría de vulnerabilidad, se utilizaron los libros rojos de mamíferos, aves y reptiles de Colombia (Castaño-Mora, 2002; Renjifo, et al., 2002; RodríguezMahecha, et al., 2006). Además, después de un ejercicio de intercambio de observaciones sobre la metodología con el comité técnico del Subsistema, se adicionaron algunas especies de peces, con lo que se determinaron, finalmente, 28 especies candidatas entre mamíferos, aves y peces (Tabla 1S, https://www.raccefyn.co/index.php/raccefyn/article/ downloadSuppFile/719/3526).

A continuación, se presenta el proceso de gestión de la información seguido para cada uno de los cinco criterios y las especies candidatas.

\section{Criterio 1. Heterogeneidad.}

Parámetros de selección. Tipos de "hábitat". A partir de la clasificación de ecosistemas hecha para determinar las prioridades de conservación en el Subsistema (Ríos-Franco
\& Valencia, 2014), la cual relaciona 188 tipos diferentes de ecosistemas, se escogieron 19 de ellos (Tabla 1), los cuales resultaron de unir aquellos que representaban un mismo tipo de cobertura y piso bioclimático, ya que no se consideró que una mayor diferenciación generara un efecto en términos de representatividad para alguna de las especies candidatas. Por ejemplo, los bosques de páramo en la clasificación original se dividían en pluviales, muy húmedos, húmedos o secos y, además, por cordilleras (3) y por vertientes (2), lo que resultaba en hasta 24 tipos diferentes de ecosistemas para cada tipo de bosque y piso bioclimático.

Para cada uno de los 19 tipos de ecosistema, se determinó su área de ocupación en el paisaje y se especificó el valor de nivel de uso necesario para considerarlo como valor objeto de conservación (Tabla 1). En cuanto a los tipos de "hábitat", se consideró que debían incluirse cuando el nivel de uso fuera de dos o más en una escala de 0 a 3 (ver criterios de selección).

Zonas de manejo. El Subsistema de los Andes Occidentales (SAO) está divido en cuatro subsistemas temáticos: Sistema Departamental de Áreas Protegidas de Antioquía (SIDAP Antioquia), Sistema Regional de Áreas Protegidas del Eje Cafetero (SIRAP Eje Cafetero), SIRAP Macizo y SIDAP Nariño, los cuales tienen diferentes estructuras, tipos

Tabla 1. Ecosistemas naturales remanentes de la territorial Andes Occidentales y el nivel de representación del VOC para cada tipo de hábitat según los criterios de selección.

\begin{tabular}{rlrc}
\hline No. & Tipo de ecosistema & Área (ha) & $\begin{array}{r}\text { Nivel de uso } \\
\text { del VOC }\end{array}$ \\
\hline 1 & Arbustal andino & 1.117 .774 & 2 \\
\hline 2 & Arbustal basal & 539.727 & 2 \\
\hline 3 & Arbustal de páramo & 184.129 & 2 \\
\hline 4 & Arbustal subandino & 277.828 & 2 \\
\hline 5 & Bosque andino & 1.142 .389 & 2 \\
\hline 6 & Bosque basal & 1.827 .898 & 2 \\
\hline 7 & Bosque de páramo & 371.586 & 2 \\
\hline 8 & Bosque subandino & 960.723 & 2 \\
\hline 9 & Cuerpo de agua andino & 4.753 & 2 \\
\hline 10 & Cuerpo de agua basal & 24.120 & 2 \\
\hline 11 & Cuerpo de agua de páramo & 756 & 2 \\
\hline 12 & Cuerpo de agua subandino & 73 & 2 \\
\hline 13 & Herbazal andino & 41.761 & 2 \\
\hline 14 & Herbazal basal & 258.863 & 2 \\
\hline 15 & Herbazal de paramo & 357.542 & 2 \\
\hline 16 & Herbazal subandino & 111.853 & 2 \\
\hline 17 & Humedal basal & 115.305 & 2 \\
\hline 18 & Humedal subandino & 707 & 2 \\
\hline 19 & Ríos & 80.445 & 2 \\
\hline & & & \\
\hline
\end{tabular}

VOC: valores objeto de conservación 
de gobernabilidad, posicionamiento y estados de avance en términos de la consolidación e implementación de los planes de acción. Estos cuatro escenarios se concibieron como los factores políticos y de gestión determinantes para abordar el manejo (planificación e implementación de las acciones y el monitoreo) en los diferentes sectores del paisaje. En este sentido, se estableció que los cuatro subsistemas temáticos serían las zonas de manejo que debían quedar representadas por los valores objeto de conservación, se determinó su área y se les asignó el valor de nivel de uso con el cual quedarían representados como valor objeto de conservación (Tabla 2). Asimismo, se consideró que las zonas de manejo quedaban representadas cuando el nivel de uso fuera de 2 o más en una escala de 0 a 3 (ver criterios de selección).

Criterios de selección. Se determinó qué tanto representaba cada una de las especies candidatas a los 19 ecosistemas y a las cuatro zonas de manejo, así: " $0=\mathrm{La}$ población de la especie candidata no usa este tipo de hábitat o zona de manejo. Esta especie, o nunca ha sido vista allí, o solo se la ha visto de manera ocasional (por ejemplo, un animal explorando este terreno). 1= La población de la especie candidata usa este tipo de hábitat o zona de manejo. Sin embargo, dada la abundancia y persistencia de esta especie, es muy poco probable que sufra un fuerte impacto si se produce una disminución en la extensión, calidad o acceso al hábitat o zona de manejo. La especie puede compensar fácilmente esas reducciones haciendo uso de otros hábitats o zonas. $2=$ La población de la especie candidata es altamente dependiente de este tipo de hábitat o zona de manejo, aunque no requiere de él para su persistencia. Una reducción en la extensión, calidad o acceso a este hábitat o zona de manejo tendrá impactos significativos sobre la abundancia y la distribución de la especie candidata en el paisaje; sin embargo, la perdida de este muy probablemente no causará su extinción local. Este valor puede considerarse como una selección apropiada si las observaciones de una especie indican que pasa una cantidad considerable de tiempo en el hábitat o zona de manejo, aunque no quede claro si es que técnicamente "requiere" de este. $3=$ La población de una especie candidata requiere este tipo de hábitat o zona de manejo para cumplir con su ciclo de vida. Si el hábitat o zona de manejo es destruido, la especie se extinguirá localmente." (Stringberg, 2007).

\section{Criterio 2. Área}

Parámetros de selección. Se definió que el rango de hogar se registraría con valores continuos.

Criterios de selección. Además de la estimación del rango de hogar para cada especie, se tuvo en cuenta si había distancias de dispersión largas ( $>$ a $10 \mathrm{~km})$, si la conectividad (calificados como "SI" o "No") y la proporción de área ocupada por la especie en el paisaje eran importantes. Para incluir la estimación del rango de hogar se revisaron publicaciones que documentaran estudios para cada especie y se consultaron expertos de los diferentes grupos (Tabla 1S). Para determinar la proporción de área del paisaje
Tabla 2. Zonas de manejo de la Territorial Andes occidentales y el nivel de representación del VOC para cada una según los criterios de selección.

\begin{tabular}{clcc}
\hline No. & Zonas de manejo & Área (ha) & Nivel de uso del VOC \\
\hline 1 & Antioquia & 6.296 .422 & 2 \\
2 & Eje cafetero & 3.196 .752 & 2 \\
3 & Macizo & 4.810 .684 & 2 \\
4 & Nariño & 3.507 .300 & 2 \\
\hline
\end{tabular}

VOC: valores objeto de conservación

ocupada por cada especie candidata, se ajustaron los polígonos de distribución de la Unión Internacional para la Conservación de la Naturaleza (UICN), teniendo en cuenta el rango altitudinal de localización de la especie. Por ejemplo, para el mono aullador rojo, el polígono de la UICN se extiende desde el borde oriental de la región Pacífico hasta la Amazonia brasilera, incluidas las partes más altas de las cordilleras Central y Oriental (>5.000 m). La presencia del mono aullador se ha confirmado solo hasta los 3.200 msnm (Hernández-Camacho \& Cooper, 1976), por lo tanto, el área por encima de dicha altitud se sustrajo del polígono. Posteriormente se calculó la proporción de la Dirección Territorial Andes Occidentales con respecto al área de distribución de la especie. La gestión de la información se llevó a cabo en el laboratorio de Sistemas de Información Geográfica de la Dirección Territorial Andes Occidentales de Parques Nacionales Naturales de Colombia con el programa ArcGis 9.2.

\section{Criterio 3. Vulnerabilidad}

Parámetros de selección. Para definir la vulnerabilidad de cada especie de paisaje, se tuvieron en cuenta las actividades humanas que se hubieran convertido o pudieran llegar a convertirse en una amenaza para la conservación de la biodiversidad o los servicios ecosistémicos. Queda implícito que no toda actividad humana es necesariamente una amenaza, ya que ello depende de la gravedad, la proporción del área afectada y la resiliencia del sistema (especie, comunidad, etc.). Se definieron 15 actividades humanas: tala para cultivos y ganadería, quema, extracción de madera, y flora no maderable, cacería por conflicto, consumo y comercio, introducción de especies animales y vegetales, minería, construcción de vías y represas, disposición de residuos en áreas de conservación y ecoturismo. Asimismo, para cada actividad humana se determinó la urgencia y la probabilidad de que ocurriera. "La urgencia define cuán pronto ocurrirá una actividad humana en particular: $0=\mathrm{Si}$ ocurre no lo hará en los próximos 10 años. $1=\mathrm{Si}$ ocurre, podría ser en los próximos 3 a 10 años. $2=$ Si ocurre, podría ser en los próximos 1 a 3 años. 3= La amenaza ya está ocurriendo y se debe actuar de inmediato. La probabilidad de que la actividad ocurra es el grado de incertidumbre asociado con una actividad humana particular y se determina 
con un valor entre 0 y 1 (por ejemplo, si se piensa que hay un $25 \%$ de probabilidad de que se construya una represa, se debe ingresar 0,25)." (Stringberg, 2007). Todas las actividades humanas relacionadas en la Dirección Territorial Andes Occidentales obtuvieron una calificación de 3 en cuanto a la urgencia y de 1 en cuanto a la probabilidad de que ocurriera.

Criterios de selección. La vulnerabilidad de cada espe-cie candidata en el paisaje se determina a partir de su estatus de conservación (Castaño-Mora, 2002; Renjifo, et al., 2002; Rodríguez-Mahecha, et al., 2006) y un índice de amenaza que integra la gravedad con que cada actividad humana afecta la especie, la capacidad de recuperación de esta una vez se elimina esa actividad humana, y la proporción de área que ocupa la especie en el paisaje afectado por dicha actividad humana. "La gravedad es la medida en que una amenaza particular disminuye la abundancia y la distribución local de la especie, así: $0=$ Ninguna $o$ positiva. 1= Poca, es decir, un efecto medible, aunque pequeño sobre la densidad o la distribución. 2= Alguna, por su efecto sustancial sobre la densidad o la distribución, aunque la erradicación local es poco probable. $3=$ Seria, ya que la erradicación local es probable." (Stringberg, 2007). El tiempo de recuperación se refiere a "cuán rápido la población puede recuperarse de los impactos de una amenaza particular (que está ocurriendo o puede ocurrir) si es que esta es eliminada. El tiempo de recuperación se define seleccionando uno de los siguientes valores: $0=$ Inmediato o en menos de 1 año. $1=$ Recuperación entre 1 y 10 años. $2=$ Recuperación entre 10 y 100 años. $3=\mathrm{La}$ recuperación puede tomar más de 100 años o simplemente puede que no ocurra. La proporción de área afectada es la proporción estimada de la distribución local de la especie (en el paisaje) que es afectada por una amenaza particular, así: $0=$ La distribución local no se ve afectada. $1=$ Menos del $10 \%$ de la distribución local se ve afectada. $2=10$ a 25 $\%$ de la distribución local se ve afectada. $3=25$ a $50 \%$ de la distribución local se ve afectada. 4= Más de la mitad de la distribución local se ve afectada." (Stringberg, 2007).

Para determinar la proporción del área afectada por cada actividad humana y cada especie candidata, se usó la metodología de CORINE Land Cover adaptada para Colombia, escala 1: 100.000 (Ideam, 2010), recurriendo a la función "Clip" de la Shape Management Tool del Arc Gis 9,2. Todas las especies candidatas se calificaron según todas las actividades humanas y los tres criterios (Tablas 2S, https://www.raccefyn.co/index.php/raccefyn/article/ downloadSuppFile/719/3527, 3S, https://www.raccefyn. co/index.php/raccefyn/article/downloadSuppFile/719/3528 y 4S, https://www.raccefyn.co/index.php/raccefyn/article/ downloadSuppFile/719/3529).

\section{Criterio 4. Funcionalidad}

Parámetros de selección. Se relacionaron 10 funciones ecológicas que dan lugar en el paisaje de la territorial Andes Occidentales: herbívora, depredación de primer, segundo y mayor orden, degradación de materia orgánica, depredación y dispersión de semillas, polinización, disturbio mecánico, control de plagas e interacción competitiva.

Criterios de selección. Cada especie candidata se calificó según la importancia de su papel con respecto a cada una de las funciones ecológicas consideradas, así: " $0=$ La especie no desempeña ningún papel en esa función. $1=$ Se piensa que la especie tiene algún efecto o tiene poco efecto. $2=\mathrm{La}$ especie tiene un efecto claro. 3= La especie tiene un efecto fuerte" (Stringberg, 2007) (Tabla 5S, https://www.raccefyn. co/index.php/raccefyn/article/downloadSuppFile/719/3530).

\section{Criterio 5. Importancia socioeconómica}

Parámetros de selección. Para determinar la importancia socioeconómica de cada especie candidata en el paisaje, se consideró si tenía un valor económico positivo o negativo y un valor cultural positivo o negativo (una misma especie en el paisaje puede tener valores culturares y económicos tanto positivos como negativos). Además, se consideró su potencial como especie emblemática o bandera.

Criterios de selección. Cada especie fue calificada según las cinco opciones, así: " $0=$ Especie sin significación. 1= Especie con alguna significación. 2= Especie con gran significación.” (Stringberg, 2007) (Tabla 3).

Posteriormente se calcularon los puntajes originales y normalizados para cada criterio y el puntaje agregado para cada especie (para los detalles de las ecuaciones, ver el Manual técnico 5 del Living Landscapes Program de Stringberg, 2007) (Tabla 6S, https://www.raccefyn.co/ index.php/raccefyn/article/downloadSuppFile/719/3531).

Análisis de datos. Antes de iniciar la selección de los valores objeto de conservación, se definieron los siguientes parámetros: el margen de error para seleccionar especies con puntajes agregados similares; el valor de corte para definir cuándo una amenaza individual estaba representada por una especie dentro del conjunto final del valor objeto de conservación, y el valor mínimo requerido del estatus de conservación. El margen de error y el valor de corte para que una amenaza quedara representada fueron de $5 \%$ y 0,5 , respectivamente. Además, dado que todas las especies candidatas estaban clasificadas bajo algún criterio de amenaza, todas se incluyeron en la categoría de estatus de conservación.

En el proceso de selección final de los valores objeto de conservación participaron los comités técnicos de los subsistemas y se usó el programa Landscape species 2.0 como herramienta de apoyo para los cálculos y las diferentes iteraciones. En cuanto a la selección de los valores objeto de conservación del subsistema, se escogió la especie con el mayor puntaje agregado. Al escoger la primera especie se extrajeron los tipos de "hábitat", las zonas de manejo, las actividades humanas y las funciones ecológicas que representaba.

A continuación, se escogió la especie con el puntaje agregado más alto que representara nuevos tipos de hábitat, zonas de manejo, actividades humanas o funciones 
Tabla 3. Calificación de la importancia socioeconómica de cada especie candidata

\begin{tabular}{|c|c|c|c|c|c|}
\hline \multirow[t]{2}{*}{ Nombre común } & \multirow[t]{2}{*}{ Potencial especie bandera } & \multicolumn{2}{|c|}{ Valor cultural local } & \multicolumn{2}{|c|}{ Valor económico } \\
\hline & & Positivo & Negativo & Positivo & Negativo \\
\hline Mono aullador rojo & 2 & 1 & 0 & 1 & 0 \\
\hline Pato colorado & 1 & 1 & 1 & 1 & 1 \\
\hline Mono araña negro & 2 & 2 & 0 & 2 & 0 \\
\hline Mono araña café & 2 & 1 & 0 & 1 & 0 \\
\hline Lorito cadillero & 1 & 0 & 0 & 0 & 0 \\
\hline Tangara multicolor & 1 & 1 & 0 & 0 & 0 \\
\hline Paujil de pico azul & 2 & 2 & 0 & 2 & 0 \\
\hline Guagua loba & 2 & 2 & 0 & 2 & 0 \\
\hline Cacique candela & 1 & 0 & 0 & 0 & 0 \\
\hline Mono churuco & 2 & 2 & 0 & 2 & 0 \\
\hline Perico paramuno & 2 & 1 & 0 & 0 & 0 \\
\hline Nutria & 2 & 0 & 1 & 2 & 2 \\
\hline Loro orejiamarillo & 2 & 1 & 0 & 1 & 0 \\
\hline Águila crestada & 2 & 0 & 2 & 0 & 2 \\
\hline Jaguar & 2 & 1 & 1 & 0 & 2 \\
\hline Pava caucana & 2 & 1 & 0 & 2 & 0 \\
\hline Zambullidor plateado & 1 & 0 & 0 & 0 & 0 \\
\hline Puma & 2 & 0 & 0 & 0 & 2 \\
\hline Danta centroamericana & 2 & 2 & 0 & 2 & 0 \\
\hline Danta común & 2 & 2 & 0 & 2 & 0 \\
\hline Danta de páramo & 2 & 2 & 0 & 2 & 0 \\
\hline Oso de anteojos & 2 & 0 & 2 & 0 & 2 \\
\hline Cóndor & 2 & 2 & 2 & 0 & 0 \\
\hline Sabaleta & 0 & 0 & 0 & 2 & 0 \\
\hline Mojarra negra & 0 & 0 & 0 & 2 & 0 \\
\hline Bagre blanco & 0 & 0 & 0 & 2 & 0 \\
\hline Mico tití gris & 2 & 0 & 0 & 0 & 2 \\
\hline Manatí & 2 & 2 & 0 & 2 & 0 \\
\hline
\end{tabular}

ecológicas. Una especie puede tener un puntaje agregado muy alto, pero si no representa nuevos elementos con relación a una especie previamente seleccionada, no se la tiene en cuenta como valor objeto de conservación para evitar la redundancia de los esfuerzos de conservación en el paisaje.

Cuando varias especies presentaron un valor agregado que no se diferenciaba significativamente en cuanto al margen de error predefinido, se evaluó cuál especie presentaba mayores oportunidades de gestión y esta se seleccionaba.

\section{Resultados}

En cuatro ejercicios iterativos llevados a cabo por el equipo técnico de la Dirección Territorial Andes Occidentales (DTAO) y en algunos subsistemas temáticos se seleccionaron como valores objeto de conservación el oso, el puma, el pato colorado y el manatí, siendo el oso el primero en todos los resultados. La danta de páramo y el jaguar se seleccionaron en tres de los cuatro ejercicios. Además, el mono churuco se seleccionó en dos análisis, en tanto que la nutria y la guagua loba se seleccionaron cada uno en una de las iteraciones (Tabla 4).

\section{Discusión}

El oso andino depende principalmente de los bosques altoandinos y de páramo. Está presente en los cuatro subsistemas temáticos, lo que no lo hace dependiente exclusivamente de uno de ellos (Tabla 5), y está asociado a 12 de las 15 actividades humanas que podrían traducirse en amenazas directas para la biodiversidad en este paisaje, siendo particularmente significativo el efecto de la cacería por conflicto (Tabla 7S, https://www.raccefyn.co/index.php/ raccefyn/article/downloadSuppFile/719/3532). 
Tabla 4. Especies Valor Objeto de Conservación (VOC) seleccionadas para la Territorial Andes Occidentales.

\begin{tabular}{cllll}
\hline Orden & $\begin{array}{l}\text { Asesores en Biología de la } \\
\text { conservación de SINAP y DTAO }\end{array}$ & $\begin{array}{l}\text { Equipo técnico de } \\
\text { DTAO }\end{array}$ & $\begin{array}{l}\text { Comité técnico de } \\
\text { SIRAP Macizo }\end{array}$ & $\begin{array}{l}\text { Comité técnico de } \\
\text { SIRAP Eje Cafetero }\end{array}$ \\
\hline 1 & Oso andino & Oso andino & Oso andino & Oso andino \\
\hline 2 & Puma & Jaguar & Puma & Puma \\
\hline 3 & Pato colorado & Pato colorado & Danta de páramo & Danta de páramo \\
\hline 4 & Danta de páramo & Mono churuco & Pato colorado & Jaguar \\
\hline 5 & Jaguar & Manatí & Nutria & Pato colorado \\
\hline 6 & Mono churuco & Puma & Manatí & Guagua loba \\
\hline 7 & Manatí & & & Manatí \\
\hline
\end{tabular}

Sistema Nacional de Áreas Protegidas (SINAP)

Sistema Regional de Áreas Protegidas (SIRAP)

Dirección Territorial Andes Occidentales (DTAO)

Tabla 5. Representación de las zonas de manejo (subsistemas temáticos) para los valores objeto de conservación del Subsistema Andes Occidentales

\begin{tabular}{lcccc}
\hline VOC & Antioquia & Eje cafetero & Macizo & Nariño \\
\hline Pato colorado & 0 & 3 & 3 & 3 \\
Guagua loba & 2 & 2 & 3 & 0 \\
Mono churuco & 3 & 0 & 2 & 2 \\
Nutria & 2 & 2 & 2 & 2 \\
Jaguar & 3 & 1 & 2 & 2 \\
Puma & 2 & 2 & 2 & 2 \\
Danta de páramo & 1 & 2 & 0 & 0 \\
Oso de anteojos & 2 & 2 & 0 & 2 \\
Manatí & 3 & & & 2 \\
\hline
\end{tabular}

VOC: valores objeto de conservación

El puma es la especie con mayor representación de ecosistemas dentro del subsistema (14/19) (Tabla 5), lo cual está asociado con su amplia distribución altitudinal. Se distribuye en los cuatro subsistemas temáticos (Tabla 5) y es vulnerable a 11 de las 15 actividades humanas relacionadas (Tabla 7S), entre las cuales se destaca la cacería para consumo de especies que hacen parte de su dieta, lo cual degrada su hábitat.

El pato colorado representa los cuerpos de agua andinos y de páramo, no el SIDAP Antioquia, y está asociado principalmente a la introducción de especies animales, específicamente a la trucha arcoíris (Oncorhynchus mykiss) y a otras actividades que generan la degradación de su hábitat, incluida la minería.

El manatí representa exclusivamente los ecosistemas acuáticos basales únicamente en el SIDAP Antioquia, por lo tanto, dentro del SAO depende exclusivamente de la efectiva gestión ambiental en ese subsistema. Esta especie se ha visto fuertemente afectada por la introducción de especies vegetales y la cacería por consumo.
La danta de páramo representa casi todos los ecosistemas del bosque altoandino hacia arriba, incluidos los cuerpos de agua. Depende en buena parte de la efectiva gestión en todos los subsistemas, aunque en menor proporción del SIDAP Antioquia. La especie está afectada particularmente por la cacería por conflicto, asociada probablemente con el cultivo de papa, y a la cacería para consumo.

El jaguar está asociado con ocho de los diez ecosistemas por debajo de los sistemas subandinos, pero principalmente con los bosques basales. Depende fundamentalmente de la efectiva gestión en el SIDAP Antioquia, y la actividad humana que más lo afecta directamente es la cacería por conflicto, además de la reducción o degradación de los bosques basales debido a la cacería para consumo de las especies que hacen parte de su dieta.

El mono churuco depende exclusivamente de los bosques en zonas basales y subandinas, además de dos subsistemas temáticos sin continuidad, el SIDAP Antioquia y el SIRAP Macizo. La tala rasa para cultivos y pasturas y la 
quema son las actividades humanas que más lo afectan al ser una especie dependiente de doseles altos. El mono churuco también se ve afectado por la cacería para consumo.

La nutria depende de la asociación de bosques subandinos y basales con los ríos y cuerpos de agua y está en los cuatro subsistemas temáticos. Además de ser vulnerable a las actividades que generan la degradación de su hábitat, es la especie del paisaje más vulnerable a la cacería para comercio.

Por último, la guagua loba depende exclusivamente de los bosques altoandinos, desde Antioquia hasta el Macizo, y se ve afectada principalmente por la cacería para consumo y, además, por la reducción y la fragmentación de su hábitat.

Cinco de los nueve valores objeto de conservación son grandes mamíferos. Los grandes mamíferos, principalmente los grandes carnívoros, se han usado como sustitutos en diferentes categorías: como especie piedra angular (Sinclair, 2003; Ucarli, 2011), bandera (Clark, 2004; Ucarli, 2011) o sombrilla (McKelvey, et al., 2000; Ucarli, 2011). No obstante, su potencial como objeto sustituto depende del contexto asociado y qué tanto representa los objetivos de conservación y las situaciones de manejo (Sergio, et al., 2008, 2006). También se seleccionaron especies de otros niveles de la cadena alimenticia que permiten incrementar la eficacia al combinar estrategias de conservación (Hanley, $\boldsymbol{e t}$ al., 2005; Keuroghlian, et al., 2009).

El oso andino se ha usado como objeto sustituto en Ecuador desde la perspectiva de especie bandera. Sin embargo, se reconoce que se hizo pensando en un público general y no en las comunidades locales, las cuales tenían interacciones económicas negativas con la especie como resultado de la actividad depredadora de este úrsido contra el ganado bovino (Clark, 2004). Tácitamente se lo reconoce como una especie sombrilla y piedra angular que puede servir de herramienta efectiva de conservación, aunque su eficacia debe comprobarse empíricamente (Sanderson, et al., 2002). En general, los grandes felinos se han concebido como especies piedra angulares o carismáticas (McKelvey, et al., 2000) y el jaguar, por ejemplo, se ha propuesto como especie paisaje para bosques atlánticos en Brasil (Cullen Jr, 2006). Por otra parte, diversas especies de nutrias se han usado como objetos sustitutos y la nutria americana se ha propuesto explícitamente como especie sombrilla para el Neotrópico (Stevens, et al., 2011).

En Colombia y en los países vecinos existen programas o iniciativas de conservación del oso en diferentes escalas de paisaje (Arjona, et al., 2012, Boher, et al., 1994, Rodríguez, 2001, Rodríguez, et al., 2003), de la danta de montaña (Lizcano, et al., 2004), de felinos, incluidos el jaguar y el puma (Castaño-Uribe, et al., 2013) y de la guagua loba (Saavedra-Rodríguez, et al., 2012), así como propuestas de gestión que incluyen los corredores para el jaguar, el oso y el manatí planeados por Corantioquia (González-Caro, et al., 2014 ), y otros para el puma y el mono churuco están siendo contemplados por el SIDAP Antioquia, con los cuales se pretende integrar los objetivos de manejo y articular las actividades de cada estrategia.

Es claro que el tamaño usual de las áreas protegidas no alcanza para conservar poblaciones viables de la mayoría de estas especies (Noss, et al., 1996), y es en este sentido que se decidió consolidar el SINAP. Sin embargo, para definir los objetivos de manejo en esta escala de paisaje se hace necesario determinar la disponibilidad actual de hábitat de estas especies, inicialmente siguiendo métodos basados en principios fundamentales $\mathrm{y}$, posteriormente, validándolos con modelos estadísticos (Craighead \& Cross, 2007), a partir de lo cual se definen sus necesidades de conectividad (Clark, 2004). La estructura ecológica principal de este paisaje emergería de la red ecológica conformada por las áreas mínimas requeridas en cuanto a estructura y configuración para mantener poblaciones viables de las especies paisaje (Baguette, et al., 2013, Lambeck, 1997; Roberge \& Angelstam, 2004).

Esta aproximación asume que debido a sus características, estas especies son adecuadas como elementos sustitutos de la biodiversidad. Sin embargo, es necesario probar esta hipótesis verificando empíricamente la riqueza y la abundancia en grupos indicadores (Sergio, et al., 2008).

Para el caso particular de los indicadores de abundancia ideales para el monitoreo de poblaciones animales, se pueden incluir tamaños poblacionales, densidades o sustitutos según la proporción o cantidad de área usada u ocupada, asumiendo que a mayor abundancia mayor será el área usada $\mathrm{u}$ ocupada por una especie en un territorio, y, por ende, su supervivencia, fecundidad y crecimiento. El indicador seleccionado depende de la capacidad de implementar las metodologías disponibles para cada uno.

Entre las metodologías disponibles que permiten tener estimaciones más precisas, ya que permiten calcular la probabilidad de detección y el área de muestreo real, se cuenta con los modelos de ocupación (MacKenzie, et al., 2002), el muestreo por distancia (Buckland, et al., 2001), el método de marca y recaptura (Nichols, 1992), así como un método que permite estimar densidades con cámaras trampa sin necesidad del reconocimiento individual (Rowcliffe, et al., 2008).

Los modelos de ocupación estiman la proporción del área (o parches) ocupada o usada y pueden emplearse en áreas relativamente grandes, dados los recursos y la capacidad logística implícitas, y en organismos susceptibles de ser detectados con cualquier tipo de registro (directo o siguiendo rastros). Por otro lado, el muestreo por distancias conviene en áreas moderadamente grandes y organismos con abundancias relativamente altas que sean detectables por observación directa. No obstante, hay variaciones que permiten recurrir al muestreo por distancias utilizando rastros, siempre y cuando sea posible estimar la tasa de producción y de desaparición del rastro.

El método de marca y recaptura es aplicable en áreas relativamente pequeñas, con tamaños poblacionales moderados y organismos susceptibles de ser capturados, marcados, 
liberados y reconocidos durante todo el experimento. Esta metodología tiene como limitante que no estima el área de muestreo, la cual debe calcularse con base en las distancias de dispersión de algunos individuos, lo cual puede afectar la traducción del tamaño poblacional a densidades.

Por último, la estimación de la densidad mediante cámaras trampa y sin necesidad de reconocimiento individual es una metodología aplicable a varios vertebrados terrestres medianos y grandes; con ella pueden explorarse áreas inferenciales variables, pero ello implica una gran cantidad de cámaras o la capacidad de moverlas frecuentemente para obtener los tamaños de muestra adecuados.

Dado el tamaño de la DTAO y el hecho de que la mayoría de los valores objeto de conservación seleccionados eran organismos que se detectan con dificultad y cuya abundancia es relativamente baja, se planteó un plan de monitoreo para los indicadores de abundancia siguiendo el método de Mackenzie (2002), el cual permite usar cualquier tipo de registro (incluidos los tomados mediante técnicas de 'fototrampeo'). Además, se planteó implementar el método de Rowcliffe (2008) para medir la densidad poblacional y evaluar si se lograban estimaciones con la suficiente precisión para poder detectar cambios en caso de haberlos (poder estadístico).

En algunos casos las especies paisaje podrían no cobijar algunas cuya distribución geográfica es restringida y cuyos requerimientos ecológicos son específicos, o sobre las cuales hay amenazas particulares (Andelman \& Fagan, 2000; Fontaine, et al., 2007; Lindenmayer, et al., 2014). En este sentido, es necesario analizar cuáles de esos componentes de la biodiversidad podrían requerir acciones particulares, lo que complementaría la lista. Normalmente, en estas adiciones se integran especies que necesitan estrategias de conservación basadas en el desplazamiento de organismos vivos (reintroducciones, refuerzos, suplementos o introducciones) (UICN, 1998), pues sus poblaciones están tan reducidas y aisladas que el garantizar únicamente el mantenimiento o recuperación de su hábitat no garantiza la recuperación de las poblaciones.

Por lo tanto, una vez determinada la disponibilidad de hábitat y las áreas con mayor probabilidad de conectividad, se deberá consultar a los expertos de otros grupos, y a botánicos, herpetólogos, entomólogos, para evaluar cuáles organismos deben ser incluidos, con el fin de complementar los valores objeto de conservación.

\section{Conclusiones}

El presente estudio representa el primer aporte para la Dirección Territorial Andes Occidentales sobre la selección de valores objeto de conservación que pueden ser elementos sustitutos de la biodiversidad y los servicios ecosistémicos en el SAO. A partir de esta selección se hace necesario analizar la distribución del hábitat actual y futuro de dichos objetos de conservación teniendo en cuenta que está determinada por diferentes parámetros físicos y bióticos que pueden tener mayor o menor incidencia dependiendo de los requerimientos ecológicos de cada especie. Además, el conocimiento de los hábitats de dichos valores contribuye a una mejor planificación de la gestión del territorio y la conservación de las diferentes especies. Por ello, se pretende ampliar su selección y conocimiento en todo el ámbito andino para obtener información a una mayor escala.

\section{Información suplementaria}

Tabla 1S. Especies candidatas definidas para el Subsistema Andes Occidentales. Vea la tabla $1 \mathrm{~S}$ en: https://www.raccefyn.co/index. php/raccefyn/article/downloadSuppFile/719/3526

Tabla 2S. Actividades humanas: calificación según la gravedad con que cada actividad humana afecta la especie candidata. $0=$ ninguna o positiva, $1=$ poca-efecto medible aunque pequeño sobre la densidad o la distribución, $2=$ alguna - efecto sustancial sobre la densidad o la distribución, aunque la erradicación local es poco probable, $3=$ la erradicación local es probable. Vea la tabla $2 \mathrm{~S}$ en: https:/www.raccefyn.co/index.php/raccefyn/article/ downloadSuppFile/719/3527

Tabla 3S. Actividades humanas: calificación según la capacidad de recuperación de la especie una vez removida la actividad humana. $0=$ inmediato $o$ en menos de 1 año, $1=$ Recuperación entre 1-10 años, $2=$ Recuperación entre 10-100 años y 3=Recuperación puede tomar más de 100 años o simplemente puede que no ocurra. Vea la tabla $3 \mathrm{~S}$ en: https:/www.raccefyn.co/index.php/raccefyn/article/ downloadSuppFile/719/3528

Tabla 4S. Actividades humanas: proporción de área de paisaje que ocupa la especie afectada por la actividad humana. $0=\mathrm{La}$ distribución local no es afectada, $1=<10 \%$ de la distribución local es afectada, $2=10 \%-25 \%$ de la distribución local es afectada, $3=25 \%-50 \%$ de la distribución local es afectada. 4 = Más de la mitad de la distribución local es afectada. Vea la tabla 4S en: https://www.raccefyn.co/index.php/raccefyn/article/ downloadSuppFile/719/3529

Tabla 5S. Calificación de funciones ecológicas de las especies. $0=$ La especie no desempeña ningún papel en esa función, $1=$ Se piensa que la especie tiene algún efecto o tiene un efecto débil, $2=\mathrm{La}$ especie tiene un efecto claro y $3=$ La especie tiene un efecto fuerte. Vea la tabla $5 \mathrm{~S}$ en: https://www.raccefyn.co/index.php/raccefyn/ article/downloadSuppFile/719/3530

Tabla 6S. Puntajes agregados de importancia socioeconómica para casa especie candidata. Vea la tabla $6 \mathrm{~S}$ en: https://www.raccefyn. co/index.php/raccefyn/article/downloadSuppFile/719/3531

Tabla 7S. Amenazas directas para los VOC seleccionados para el Subsistema Andes Occidentales. Vea la tabla 7S en: https://www. raccefyn.co/index.php/raccefyn/article/downloadSuppFile/719/3532

\section{Agradecimientos}

Al comité técnico del Subsistema Andes Occidentales y a los comités técnicos de los Subsistemas temáticos SIDAP Antioquia, SIRAP Eje Cafetero y SIRAP Macizo, por respaldar esta iniciativa y permitir su continuidad. Al director y a todo el equipo técnico de la Dirección Territorial Andes Occidentales de Parques Nacionales Naturales de Colombia, por realimentar continuamente el 
proceso. A Samantha Stringberg, por apoyar el análisis. A Milena del Pilar Marrugo, por las sugerencias en la edición del documento y a Fernando Ayerbe Quiñones y otros especialistas de los diferentes grupos, por la información sobre las especies candidatas.

\section{Contribución de los autores}

Néstor Javier Roncancio recopiló y analizó la información primaria y secundaria, y redactó y editó el documento final. Laura Alejandra Vélez Vanegas contribuyó a la recopilación de la información secundaria, la redacción y la edición del documento final.

\section{Conflicto de intereses}

Los autores del manuscrito declaran que no tienen conflicto de intereses.

\section{Referencias}

Andelman, S. J. \& Fagan, W. F. (2000). Umbrellas and flagships: Efficient conservation surrogates or expensive mistakes? Proceedings of the National Academy of Sciences. 97 (11): 5954-5959.

Angelstam, P. K., Bütler, R., Lazdinis, M., Mikusiński, G., Roberge, J.-M. (2003). Habitat thresholds for focal species at multiple scales and forest biodiversity conservation dead wood as an example. Annales Zoologici Fennici. 40 (6): 473-482.

Arjona, F., Rodríguez-Mahecha, J.V., Restrepo H. (2012). Plan de manejo y conservación del oso andino (Tremarctos ornatus) en la juridicción de la Corporación Regional de Cundinamarca. Conservación Internacional. Colombia.

Baguette, M., Blanchet, S., Legrand, D., Stevens, V. M., Turlure, C. (2013). Individual dispersal, landscape connectivity and ecological networks. Biological Reviews of the Cambridge Philosophical Society. 88 (2): 310-326.

Boher, S., Cuesta, M., De Luca, A., Goldstein, I., Lamas, M., Lobo, A., Marín, B., Michelangeli, F., Mondolfi, E., Pernalete, J., Rodríguez, J.P., Torres, D., Waugh D., Yerena, E. (1994). Plan de acción para el oso andino en Venezuela. Grupo Ad Hoc de especialistas del oso frontino en Venezuela.

Branton, M. \& Richardson, J. S. (2011). Assessing the Value of the Umbrella-Species Concept for Conservation Planning with Meta-Analysis. Conservation Biology. 25 (1): 9-20.

Buckland, S. T., Anderson, D. R., Burnham, K. P., Laake, J. L., Borchers, D. L., Thomas, L. (2001). Introduction to Distance Sampling: Estimating Abundance of Biological Populations. Oxford University Press, Oxford.

Caro, T. M. \& O'Doherty, G. (1999). On the Use of Surrogate Species in Conservation Biology. Conservation Biology. 13 (4): 805-814.

Castaño-Mora, O. V. (2002). Libro rojo de reptiles de Colombia. Libros rojos de especies amenazadas de Colombia. Instituto de Ciencias Naturales- Universidad Nacional de Colombia, Ministerio del Medio Ambiente, Conservación Internacional Colombia. Bogotá, Colombia. 160 pp.

Castaño-Uribe, C., González-Maya, J.F., Zárrate-Charry, D., Ange-Jaramillo, C., Vela-Vargas, I.M. (2013). Plan de conservación de felinos del Caribe colombiano: los felinos y su papel en la planificación regional integral basada en especies clave. Fundación Herencia Ambiental Caribe, ProCAT Colombia, The Sierra to Sea Institute. Santa Marta, Colombia.

Clark, M. R. (2004). Using the spectacled bear as a conservation tool in the Condor Bioreserve, Ecuador. Journal of Sustainable Forestry. 18 (2-3): 223-236.

Coppolillo, P., Gómez, H., Maisels, F., Wallace, R. (2004). Selection criteria for suites of landscape species as a basis for site-based conservation. Biological Conservation. 115 (3): 419-430

Craighead, F.L., Cross, B. (2007). Identifying Core Habitat and Connectivity for Focal Species in the Interior Cedar-Hemlock Forest of North America to Complete a Conservation Area Design. USDA Forest Service Proceedings. 49: 1-16.

Cullen Jr, L. (2006). Jaguars as landscape detectives for the conservation of Atlantic Forests in Brazil. PhD Thesis, University of Kent. Canterbury, Inglaterra.

Elzinga, C. L., Salzer, D.W., Willoughby, J. W., Gibbs, J. P. (2001). Monitoring plant and animal populations. Malden, USA: Blackwell Science. 372 pp.

Feinsinger, P. (2001). Designing field studies for biodiversity conservation. The Nature Conservancy. Island Press. 215 pp.

Fleishman, E., Murphy, D. D., Brussard, P. F. (2000). A new method for selection of umbrella species for conservation planning. Ecological Applications. 10 (2): 569-579.

Fontaine, B., Gargominy, O., Neubert, E. (2007). Priority sites for conservation of land snails in Gabon: Testing the umbrella species concept. Diversity and Distributions. 13 (6): 725-734.

González-Caro, S., Restrepo, Z., Yusti, C., Botero, S., López, B., Posada, L., Peña, A., Mejía, O., Álvarez, E. (2014). Antioquia un territorio para conservar. Convenio 4600000674. Gobernación de Antioquia-Jardín Botánico de Medellín. Medellín, Colombia.

Hanley, T. A., Smith, W. P., Gende, S. M. (2005). Maintaining wildlife habitat in southeastern Alaska: Implications of new knowledge for forest management and research. Landscape and Urban Planning. 72 (1): 113-133.

Hernández-Camacho, J., Cooper, R. W. (1976). The nonhuman primates of Colombia. En National Research Council. Neotropical Primates: Field Studies and Conservation: Proceedings of a Symposium on the Distribution and Abundance of Neotropical Primates (35-69). Washington, DC: The National Academies Press.

Holling, C. S. (1978). Adaptive Environmental Assessment and Management. Chichester, Inglaterra: John Wiley \& Sons. $402 \mathrm{pp}$.

Jarro-Fajardo, E. M. C. (2011). Lineamientos técnicos para la formulación de objetivos de conservación y valores objeto de conservación. Unidad Administrativa Especial del Sistema de Parques Nacionales Naturales, Colombia.

Jenkins, D. G., Brescacin, C. R., Duxbury, C. V., Elliott, J. A., Evans, J. A., Grablow, K. R., Hillegass, M., Lyon, B. N., Metzger, G. A., Olandese, M. L., Silvers, G. A., Suresch, H. N., Thompson, T. N., Trexler, C. M., Williams, N. C., Williams, S. E. (2007). Does size matter for dispersal distance?. Global Ecology and Biogeography. 16 (4): 415-425. 
Keuroghlian, A., Eaton, D. P., Desbiez, A. L. (2009). The response of a landscape species, white-lipped peccaries, to seasonal resource fluctuations in a tropical wetland, the Brazilian Pantanal. International Journal of Biodiversity and Conservation. 1 (4): 087-097.

Lambeck, R. J. (1997). Focal species: A multi-species umbrella for nature conservation. Conservation Biology. 11 (4): 849-856.

Lindenmayer, D. B., Barton, P. S., Lane, P. W., Westgate, M. J., McBurney, L., Blair, D., Gibbons, P., Likens, G. E. (2014). An empirical assessment and comparison of species-based and habitat-based surrogates: A case study of forest vertebrates and large old trees. PLOS ONE. 9 (2): e89807.

Lizcano, D. J., Medici, P., Montenegro, O., Carrillo, L., Camacho, A., Miller, P. S. (2004). Conservación de danta de montaña. Reporte final. UICN/SSC Conservation Breeding Specialist Group, Apple Valley, MN, USA.

Lyons, J. E., Runge, M. C., Laskowski, H. P., Kendall, W. L. (2008). Monitoring in the context of structured decisionmaking and adaptive management. The Journal of Wildlife Management. 72 (8): 1683-1692.

MacKenzie, D. I., Nichols, J. D., Lachman, G. B., Droege, S., Andrew Royle, J., Langtimm, C. A. (2002). Estimating site occupancy rates when detection probabilities are less than one. Ecology. 83 (8): 2248-2255.

Marcot, B. G., Flather, C. H. (2007). Species-level strategies for conserving rare or little-known species. En M.G. Raphael y R. Molina (Eds.), Conservation of Rare or Little-Known Species: Biological, Social, and Economic Considerations (125-164). Washington, DC, USA: Island Press.

McKelvey, K. S., Aubry, K. B., Agee, J. K., Buskirk, S. W., Ruggiero, L. F., Koehler, G. M. (2000). Lynx conservation in an ecosystem management context. 15: 419-441.

Nekaris, K. A.-I., Arnell, A. P., Svensson, M. S. (2015). Selecting a conservation surrogate species for small fragmented habitats using ecological niche modelling. Animals. 5 (1): 27-40.

Nichols, J. D. (1992). Capture-Recapture Models. BioScience. 42 (2): 94-102.

Noss, R., Quigley, H., Hornocker, M., Merrill, T., Paquet, P. (1996) Conservation biology and carnivore conservation in the Rocky Mountains. Cons. Biol. 10: 949-963.

Rasmussen, K. (2014). Recommendations for the Identification and Selection of Vertebrate Umbrella Species for Conservation Planning in Terrestrial Ecosystems. Master Thesis. University of Calgary. Calgary, Canada. 102 pp.

Renjifo, L., Franco M, A., Amaya E, J., Kattan, G., López L, B. (2002). Libro Rojo de Aves de Colombia. Libros rojos de especies amenazadas de Colombia. Instituto de Investigación de Recursos Biológicos Alexander von HumboldtMinisterio del Medio Ambiente. Bogotá, Colombia. 562 pp.

Ríos-Franco, C. A., Valencia, A. (2014). Informe Convenio 506/ 2013, Carder- WCS. Pereira, Colombia.

Roberge, J.-M., Angelstam, P. E. R. (2004). Usefulness of the umbrella species concept as a conservation tool. Conservation Biology. 18 (1): 76-85.

Rodríguez, D. (2001). Programa nacional para la conservación y recuperación del oso andino (Tremarctos ornatus), especie amenazada de los ecosistemas andinos colombianos. Ministerio del Medio Ambiente. Subdirección de Ecosistemas. Colombia.
Rodríguez, D., Cuesta, F., Goldstein, I., Bracho, A.E., Naranjo, L.G., Hernández O.L. (2003). Estrategia ecorregional para la conservación del oso andino en los Andes del Norte. WWF Colombia, Fundación Wii, EcoCiencia, Wildlife Conservation Society. Bogotá, Colombia. 38 pp.

Rodríguez-Mahecha, J. V., Mendoza, C. L., Nash, S. D. (2006). Libro rojo de los mamíferos de Colombia. Libros rojos de especies amenazadas de Colombia. Conservación Internacional Colombia - Ministerio del Medio Ambiente, Vivienda y Desarrollo Territorial. Bogotá, Colombia. 430 pp.

Rondinini, C., Rodríguez, A. S. L., Boitani, L. (2011). The key elements of a comprehensive global mammal conservation strategy. Philosophical Transactions of the Royal Society of London B: Biological Sciences. 366 (1578): 2591-2597.

Rowcliffe, J. M., Field, J., Turvey, S. T., Carbone, C. (2008). Estimating animal density using camera traps without the need for individual recognition. Journal of Applied Ecology. 45 (4): 1228-1236.

Saavedra-Rodríguez, C.A., Oshbar, K., Rojas, V., Roncancio, N.J., Ríos-Franco, C.A., Gutiérrez-Chacón, C., GómezPosada, C., Giraldo, P.A., Velasco, J.A. (2012). Plan de conservación y manejo de la guagua loba (Dinomys branikii) para el Sistema Regional de Áreas Protegidas del Eje Cafetero (SIRAP-EC), Colombia. Corporación Autónoma de Risaralda (CARDER). Corporación Autonoma de Caldas (CORPOCALDAS)

Sanderson, E. W., Redford, K. H., Vedder, A., Coppolillo, P. B., Ward, S. E. (2002). A conceptual model for conservation planning based on landscape species requirements. Landscape and Urban Planning. 58 (1): 41-56.

Sattler, T., Pezzatti, G. B., Nobis, M. P., Obrist, M. K., Roth, T., Moretti, M. (2014). Selection of multiple umbrella species for functional and taxonomic diversity to represent urban biodiversity. Conservation Biology: The Journal of the Society for Conservation Biology. 28 (2): 414-426.

Sergio, F., Caro, T., Brown, D., Clucas, B., Hunter, J., Ketchum, J., McHugh, K., Hiraldo, F. (2008). Top predators as conservation tools: Ecological rationale, assumptions, and efficacy. Annual Review of Ecology, Evolution, and Systematics. 39 (1): 1-19.

Sergio, F., Newton, I., Marchesi, L., Pedrini, P. (2006). Ecologically justified charisma: Preservation of top predators delivers biodiversity conservation. Journal of Applied Ecology. 43 (6): 1049-1055.

Sinclair, A. R. E. (2003). The role of mammals as ecosystem landscapers. Alces. 39: 161-177.

Stevens, S. S., Organ, J. F., Serfass, T. L. (2011). Otters as flagships: Social and cultural considerations. In Proceedings of Xth International Otter Colloquium, IUCN Otter Specialist Group Bulletin A. 28: 150-161.

Stringberg, S. (2007). Manual técnico 5: Una guía rápida de referencias para el software Selección de Especies Paisaje versión 2.1. Living Landscapes Program. Wildlife Conservation Society.

Ucarli, Y. (2011). Usability of large carnivore as a keystone species in Eastern Black Sea Region, Turkey. African Journal of Biotechnology. 10 (11): 2032-2036.

UICN. (1998). Guías para reintroducciones de la UICN. Preparadas por el Grupo Especialista en Reintroducción de la Comisión de Supervivencia de Especies de la UICN, UICN, Gland, Suiza \& Cambridge, Reino Unido. 
Western, D. (1987). Africa's elephants and rhinos: Flagships in crisis. Trends in Ecology \& Evolution. 2 (11): 343-346.

Wilcox, B. A. (1984). In situ conservation of genetic resources: Determinants of minimum area requirements. National Parks, Conservation and Development: The Role of Protected Areas in Sustaining Society. Smithsonian Institution Press, Washington, DC. p. 639-647.

Wildlife Conservation Society. (2001). Boletín 2: Las especies paisaje para la conservación basada en un sitio. Living Landscapes Program.
Wildlife Conservation Society. (2002). Boletín 3: Los papeles que cumplen las especies paisajes en la conservación basada en un sitio. Living Landscapes Program.

Wildlife Conservation Society. (2002). Boletín 4: La selección de especies paisaje. Living Landscapes Program.

Zambrano, H. (2010). Objetivos de conservación de las áreas protegidas. Cómo definirlos y cómo asociar objetos de conservación. Subdirección técnica. Unidad Administrativa Especial del Sistema de Parques Nacionales Naturales. 